\title{
DaF an öffentlichen Schulen am Beispiel eines Projekts in Rio de Janeiro
}

\author{
[German as a Foreign Language in public schools: a case study from Rio de Janeiro] \\ http://dx.doi.org/10.11606/1982-883725451
}

\author{
Mergenfel $\bigvee a z$ Ferreira $^{1}$ \\ Gabriela Marques-Schäfer ${ }^{2}$ \\ Ebal Bolacio Filho ${ }^{3}$
}

\begin{abstract}
The main objective of this article is to provide an overview of the emergence of a teaching project in the city of Rio de Janeiro to promote multilingualism in public schools. To this end, the article first draws on some official documents - such as the text of the National Framework Curriculum (BNCC) and the Guiding Principles for the Bilingual Schools Program in the City of Rio de Janeiro, which guide the teaching of foreign languages in Brazil and in the City of Rio de Janeiro. In addition, this paper presents results of a study conducted with teachers who have been working there as German teachers since the beginning of the project. The results of the study show that critical literacy and intercultural learning are fundamental principles in both teachers' perceptions and practices. Finally, this paper shows the importance of this teaching project in promoting a richer and plural education for students.
\end{abstract}

Keywords: foreign language teaching in elementary school; intercultural learning; critical literacy.

Zusammenfassung: Das Hauptziel dieses Artikels ist es, einen Überblick über die Entstehung eines Lehrprojekts der Stadt Rio de Janeiro zur Förderung der Mehrsprachigkeit an öffentlichen Schulen zu geben. Zu diesem Zweck stützt sich der Artikel zunächst auf einige offizielle Dokumente - wie z.B. den Text des Nationalen Rahmenlehrplans (BNCC) und die Leitprinzipien für das Programm für zweisprachige Schulen in der Stadt Rio de Janeiro, die das Lehren von Fremdsprachen in Brasilien und in der Stadt Rio de Janeiro leiten. Darüber hinaus werden in diesem Beitrag Ergebnisse einer Studie vorgestellt, die mit den Lehrkräften durchgeführt wurde, die seit Beginn des Projekts als Deutschlehrende dort arbeiten. Die Ergebnisse der Studie zeigen, dass critical literacy und interkulturelles Lernen grundlegende Prinzipien sowohl in der Wahrnehmung als auch in der Praxis der Lehrenden sind. Schließlich zeigt der vorliegende Beitrag, wie wichtig diese Lehrprojekt zur Förderung einer reicheren und pluralen Bildung von Schüler*innen ist.

\footnotetext{
${ }^{1}$ Universidade Federal do Rio de Janeiro, Departamento de Letras Anglo-Germânicas, Av. Horácio de Macedo, 2151, Rio de Janeiro, RJ, 22280002, Brasil. E-mail: megchenvaz@yahoo.com. ORCID: 00000002-6478-8066

${ }^{2}$ Universidade do Estado do Rio de Janeiro, Departamento de Letras Anglo-Germânicas, Rua São Francisco Xavier, 524, Rio de Janeiro, RJ, 20550-900, Brasil. E-mail: gabrielamarques@ yahoo.com. ORCID: 00000001-5574-2333

${ }^{3}$ Universidade Federal Fluminense, Departamento de Letras Anglo-Germânicas, R. Miguel de Frias, 9, Niterói, RJ, 24220-900. E-mail: ebolacio@gmail.com. ORCID: 0000-0002-6050-5591
} 
Stichwörter: frühes Fremdsprachenlernen; Fremdsprachenunterricht in der Grundschule; interkulturelles Lernen; critical literacy.

\section{Einführung}

Im Zuge der Internationalisierung der Institutionen ist das Wissen mindestens einer Fremdsprache eine Schlüsselqualifikation, um nicht nur mehr Chancen auf dem Arbeitsmarkt zu haben, sondern auch, um erfolgreich an einem Austausch mit Menschen aus verschiedenen Kulturen teilzunehmen. Aus diesen Gründen sind brasilianische Autoren wie Schlatter (2009), Schlatter \& Garcez (2012), Leffa (2014) und MarquesSchäfer \& Stanke (2021) der Annahme, dass das Angebot an Fremdsprachen in Schulen und Universitäten in Brasilien erweitert und verbessert werden muss. Das ist jedoch nicht einfach, denn das Lehren und Lernen von Fremdsprachen an öffentlichen Schulen ${ }^{4}$ in Brasilien von zahlreichen Problemen und Herausforderungen geprägt sind (LIMA 2011, LEFFA 2011, ALMEIDA 2014). Es gibt unzählige Hindernisse, die hinter dieser Schwierigkeit stecken. Zum Teil können wir dieses Szenario besser verstehen, wenn wir die nationale Sprachenpolitik verfolgen, die den Fremdsprachenunterricht in Brasilien im öffentlichen Schulwesen historisch geleitet hat. Dies hatte zur Folge, dass nur wenige Kinder im Grundschulalter die Möglichkeit haben, eine Fremdsprache zu lernen, indem sie auf eine private zweisprachige Schule oder eine Schule mit einem fremdsprachlichen Schwerpunkt gehen. In den meisten Fällen müssen die Eltern in diesen Schulen ein sehr hohes Schulgeld zahlen.

Um eine Lösung für dieses Problem zu finden, gründete die Stadt Rio de Janeiro im Jahr 2013 ein bilinguales Projekt an den öffentlichen Schulen für die Sprachen Englisch, Französisch und Spanisch. 2018 wurde das Projekt erweitert und die Sprache Deutsch wurde in drei Schulen eingeführt. Der Schwerpunkt des Sprachunterrichts an diesen Schulen liegt laut den Dokumenten (RIO DE JANEIRO 2018a; 2018b), die ihn leiten ${ }^{5}$, auf Ansätzen, die sich hauptsächlich auf Literacy-Praktiken (literacy practices) und auf die Förderung der Entwicklung der interkulturellen Kompetenz der Schüler*innen konzentrieren.

\footnotetext{
${ }^{4}$ Im Vergleich zu den öffentlichen Schulen können Privatschulen aufgrund ihrer finanziellen Situation in der Regel mehr Aktivitäten, wie z.B. zwei oder drei Fremdsprachen, außerhalb des regulären Curriculums, anbieten.

${ }^{5}$ Diese Dokumente werden in den folgenden Abschnitten des Artikels genauer vorgestellt.
} 
FERREIRA, M.V.; MARQUES-SCHÄFER, G.; BOLACIO FILHO, E. - DaF an öffentlichen Schulen

Ziel dieses vorliegenden Beitrags ist es, Ergebnisse einer Studie ${ }^{6}$ zu präsentieren, die mit Deutschlehrer*innen eines neuen Projekts zur Einführung der deutschen Sprachen in öffentlichen Schulen in Rio de Janeiro entwickelt wurden. Dabei wird in erster Linie der Versuch unternommen, folgende Forschungsfrage $\mathrm{zu}$ beantworten: Wie kann der Deutschunterricht an öffentlichen Schulen zur Entwicklung von kritischer Literacy sowie von kommunikativer interkultureller Kompetenz beitragen? Bevor die Studie und ihre Methode vorgestellt werden, werden im nächsten Kapitel ein Überblick über die Situation des Angebots an Fremdsprachenunterricht in Brasilien sowie eine Beschreibung des aktuellen Szenarios in der Stadt Rio de Janeiro dargestellt, wobei der Schwerpunkt stärker auf das neue Fremdsprachen-Projekt der Stadt Rio gelegt wird. Darüber hinaus werden grundlegende Begriffe wie critical literacy und interkulturelle Kompetenz in Bezug auf den hier untersuchten Kontext definiert und diskutiert.

\section{Theoretischer Hintergrund}

\subsection{Zum Fremdsprachenunterricht an öffentlichen Schulen in Brasilien}

Erst im 19. Jahrhundert - mit der Ankunft der königlichen Familie von Portugal in Rio de Janeiro und der Gründung des Colégio Pedro II im Jahre 1837 - begann in Brasilien der Unterricht moderner Fremdsprachen (vgl. MULIK 2012). Im Laufe des 20. Jahrhunderts gab es unterschiedliche Momente hinsichtlich der Rolle und Präsenz der Fremdsprache(n) in der Grundbildung (Ensino Fundamental). Zuerst befasste sich die Bildungsreform von 1933 mit dem Unterricht moderner Fremdsprachen: Französisch, Englisch und Deutsch an den Gymnasien (OLIVEIRA 2015). Latein war damals die einzige klassische Sprache im Lehrplan. Es kam zu einer wichtigen Änderung in der Methodik des Fremdsprachenunterrichts, indem man zur direkten Methode überging, ohne jedoch die Stundenzahl dieser Sprachen zu erhöhen, sondern nur die Stundenzahl des Lateinischen zu verringern (LEFFA 1999). Die Capanema-Reform von 1942 verstärkte einige der Ideen der vorangegangenen Reform. Mit den Worten von Day (2012):

Dies war die Reform, die dem Fremdsprachenunterricht in der Republik Brasilien die größte Bedeutung beimaß. Von der Grundschule bis zum Gymnasium (naturwissenschaftlich oder klassisch) wurden neben Latein auch Französisch, Englisch und Spanisch unterrichtet.

\footnotetext{
${ }^{6}$ Diese Studie wurde im Jahr 2019 entwickelt, also vor der Krise, die durch die neue Coronavirus-Pandemie ausgelöst wurde.
}

Pandaemonium, São Paulo, v. 25, n. 45, jan.-abr. 2022, p. 1-28 
FERREIRA, M.V.; MARQUES-SCHÄFER, G.; BOLACIO FILHO, E. - DaF an öffentlichen Schulen

Das LDB (Lei de diretrizes e bases - Bildungsrahmengesetz) 1961 brachte allerdings wichtige Veränderungen für den Fremdsprachenunterricht mit sich, insbesondere die Reduzierung des Fremdsprachenunterrichts auf weniger als $2 / 3$ dessen, was es unter der Capanema-Reform gab (LEFFA 1999: 13). Das LDB von 1971 bewirkte eine noch drastischere Reduktion der Fremdsprachenlehrstunden, die durch eine Stellungnahme des Bundesrates ebenfalls sanktioniert wurde, wonach die Fremdsprache je nach den Möglichkeiten der jeweiligen Einrichtung zusätzlich angeboten werden sollte. Dies bedeutete in der Praxis, dass viele Schüler*innen während ihrer ganzen Schulzeit keinen Kontakt mit einer Fremdsprache hatten, da sie in vielen Schulen nur noch einmal pro Woche in der Oberstufe angeboten wurden.

Mit der Neufassung des LDB-Gesetzes im Jahre 1996 wurde jedoch die Wichtigkeit des Erlernens mindestens einer Fremdsprache in der Grundausbildung bekräftigt:

$\S 5^{\circ}$ - Im allgemeinen Teil des Lehrplans wird ab der fünften Klasse das Unterrichten einer modernen Fremdsprache obligatorisch, für deren Wahl die Schulgemeinschaft verantwortlich sein wird, aber im Rahmen der Möglichkeiten der Institution.

Art. 36 Der Lehrplan des Gymnasiums hat die Bestimmungen des Abschnitts I dieses Kapitels und die folgenden Richtlinien zu beachten:

III - eine moderne Fremdsprache soll als Pflichtfach aufgenommen werden, die von der Schulgemeinschaft gewählt wird, und eine zweite, auf freiwilliger Basis, innerhalb der Möglichkeiten der jeweiligen Institutionen. (BRASIL 1996).

Der Text des Nationalen Rahmenlehrplans (BNCC - Base Nacional Comum Curricular) - der am 15. Dezember 2017 verabschiedet und am 20. Dezember 2017 vom Bildungsministerium (MEC) genehmigt wurde - brachte eine radikale und sehr besorgniserregende Änderung für den Fremdsprachenunterricht mit sich, da darin Englisch als einzige Fremdsprache vorgesehen ist, die in brasilianischen Schulen obligatorisch zu unterrichten sei. Andere Fremdsprachen können unterrichtet werden, jedoch nur in Ausnahmefällen, wobei dem Spanischen der Vorzug gegeben wird. Eine Sprache, die jedoch später ihren obligatorischen Status in der Oberstufe an Gymnasien verlieren sollte, den sie durch das Gesetz 11.161 von August 2005 erreicht hatte ${ }^{7}$ :

IX - Englische Sprache, andere Fremdsprachen können als Option angeboten werden, vorzugsweise Spanisch, je nach Verfügbarkeit der Einrichtung oder des Bildungsnetzes (Resolution CNE/CEB Nr. 3/2018, Art. 11, § 4).

\footnotetext{
${ }^{7}$ Sowohl in diesem Dokument als auch in den jüngsten Staatsdokumenten wird der Begriff „Fremdsprache“ statt „Zusatzsprache“ verwendet, um die Idee der Erweiterung des Bildungsangebots in die Fachdiskussion einzuführen. (port. língua adicional statt língua estrangeira).
} 
FERREIRA, M.V.; MARQUES-SCHÄFER, G.; BOLACIO FILHO, E. - DaF an öffentlichen Schulen

Im Text des BNCC erscheint die englische Sprache nicht als „Fremdsprache“, sondern als „Lingua Franca“. In diesem Zusammenhang wird argumentiert, dass es wichtig sei, sich auf neue Vorschläge, wie das Konzept der zusätzlichen Sprache, hinzubewegen, und in diesem Sinne kommt dies in dem Dokument zum Ausdruck:

In diesem Vorschlag ist die englische Sprache nicht mehr die des "Fremden", der aus hegemonialen Ländern kommt und deren Redner als Vorbild dient. Sie ist auch keine Variante der englischen Sprache. Aus dieser Perspektive ist die Verwendung durch Sprecher auf der ganzen Welt mit unterschiedlichen sprachlichen und kulturellen Repertoires willkommen und legitimiert. (BRASIL 2018: 243)

Nach diesem historischen Rückblick auf den Fremdsprachenunterricht an öffentlichen Schulen in Brasilien ist es festzustellen, dass es am Anfang des 20. Jahrhunderts ein großes und vielfältiges Sprachenangebot in den brasilianischen Schulen gab. Dies täuscht aber nicht darüber hinweg, dass damals nur eine kleine Minderheit Zugang zu Schulen hatte. Danach erlebte man einen allmählichen Rückgang der Präsenz sowohl von modernen als auch von klassischen Sprachen - was bis heute anhält. Wenn man heute einen Blick auf die offiziellen Dokumente wirft, die die Grundausbildung in Brasilien regeln, beobachtet man eine Unsicherheit hinsichtlich der Gewährleistung einer mehrsprachigen Bildung, da es darin keine Richtlinien gibt, die auf die Förderung der Mehrsprachigkeit hinweisen. Daher ist es wichtig, ein demokratisches und breites Angebot an Fremdsprachenunterricht an öffentlichen Schulen zu fördern. Im nächsten Abschnitt des vorliegenden Beitrags wird das neue Projekt des städtlichen Schulamts von Rio de Janeiro zur Erweiterung des Sprachangebots vorgestellt.

2.2 Das Projekt "Öffentliche Bilinguale Schulen in Rio de Janeiro": Hauptrichtlinien und Ziele

Ein wichtiger Meilenstein für die Entwicklung zweisprachiger Schulprojekte in der Stadt Rio de Janeiro war die Schaffung des Programms Rio Criança Global (PRCG) im Jahr 2009. Das Hauptziel dieses Programms war die Einführung von Englischunterricht bereits ab dem 1. Schuljahr in öffentlichen Schulen der Stadt Rio. Das PRCG, mit dessen Umsetzung im zweiten Halbjahr 2010 begonnen wurde, hatte als historischen Hintergrund auch die Abhaltung der Olympischen Spiele in Rio de Janeiro, die sechs Jahre später (2016), stattfinden sollten und die einen wichtigen Anreiz für den Englischunterricht und die Vorbereitung „,neuer Generationen auf die Olympischen 
Spiele 2016“ darstellten, laut der Bildungsministerin von Rio de Janeiro, Claudia Costim $^{8}$.

Als Fortsetzung des Programms Rio Criança Global führte die Stadtverwaltung ab 2013 zweisprachige Schulen mit Englischunterricht ein. Diese Schulen zielen darauf $a b$, neben dem englischen Sprachunterricht Methoden und Praktiken einzuführen, die Portugiesisch und Englisch in ihr tägliches Leben einbeziehen. Ende 2017 gab es in Rio de Janeiro bereits 10 zweisprachige Schulen mit Englisch und eine mit Spanisch. Angesichts der guten Leistungen und positiven Ergebnisse des Projekts wurde 2018 die Zahl der zweisprachigen Schulen auf insgesamt 25 erhöht und die französische und deutsche Sprache in das zweisprachige Spektrum aufgenommen.

Was die theoretischen und leitenden Annahmen des Projektes betrifft, lässt sich ausführen, dass einer der herausragendsten Aspekte, der auch einen wichtigen Unterschied in Bezug auf die Politik des Fremdsprachenunterrichts an regulären Schulen bis dahin darstellt, die Betonung der Mündlichkeit im globalen Programm von Rio Criança Global ist. In diesem Sinne ist hervorzuheben, dass bis vor kurzem in offiziellen Dokumenten über den Sprachunterricht das Lese- bzw. Schriftverständnis als die Fähigkeiten genannt wurden, die im Fremdsprachenunterricht vorrangig behandelt werden sollten. Auf der anderen Seite werden in den Leitlinien, an denen sich die PRCG orientiert, die vier Fertigkeiten als Gegenstand des Lehrens und Lernens von Fremdsprachen hervorgehoben, wobei als grundlegendes Ziel die Förderung „eines besseren Zugangs zu globalen Informationen über das Internet und auch einer stärkeren Interaktion mit dem Publikum, das für die Spiele von Rio 2016 erwartet wird: Touristen und Delegationen“ (RIO DE JANEIRO 2010) genannt wird.

Eine andere Prämisse, von der sich das zweisprachige Projekt seit seiner Gründung leiten lässt und die einen wichtigen Differenzierungspunkt für den Fremdsprachenunterricht in regulären öffentlichen Schulkontexten darstellt, ist daher die Suche nach der Integration von Inhalten aus verschiedenen Bereichen des Lehrplans. Mit anderen Worten, die zusätzliche Sprache beinhaltet die Behandlung der transversalen Themen in jeder Schule und wird nicht nur für die Kommunikation, sondern auch für das Erlernen bestimmter Inhalte verwendet. So sind in demselben oben erwähnten Dokument

\footnotetext{
${ }^{8}$ Verfügbar in http://www.rio.rj.gov.br/web/sme/exibeconteudo?article-id=1100300 (23.01.2021). 
FERREIRA, M.V.; MARQUES-SCHÄFER, G.; BOLACIO FILHO, E. - DaF an öffentlichen Schulen

die folgenden Leitprinzipien für das Programm für zweisprachige Schulen in der Stadt

Rio de Janeiro aufgeführt:

I die Funktion und den sozialen Gebrauch der zusätzlichen Sprache für die Planung unter Berücksichtigung des Fachs/Themas der Klasse zu berücksichtigen; II die Entwicklung von Fertigkeiten für den effektiven Gebrauch der zusätzlichen Sprache sowie von Fertigkeiten in Bezug auf ein bestimmtes Wissensgebiet (je nach Fach/Thema der Klassen) anzustreben; III die Entwicklung rezeptiver und produktiver Fertigkeiten in der zusätzlichen Sprache zu fördern; IV eine diskursive Perspektive der Sprache einzunehmen und sich von der ausschließlich auf das Grammatik- und Lexikonlernen konzentrierten Arbeit zu entfernen; V das Verständnis der sozio-internationalen Natur des Lernens mit methodischen Ansätzen zu reflektieren, die Erfahrungen und Interaktionen in der zusätzlichen Sprache fördern; und VI die affektiven, kognitiven und kulturellen Aspekte des Lehr- und Lernprozesses als gleichermaßen wichtig zu behandeln (DOM-RJ 2018: 60).

Die Stellungnahme des CME (Stadtrat für Bildung, port. Conselho Municipal de Educação) enthält auch einige Klassifizierungen, die die Definition einer zweisprachigen Schule untermauern, wie z.B. "die Existenz eines Kulturabkommens zwischen Brasilien und dem Land, das der als "zweite Heimatsprache" angebotenen Sprache entspricht, die nun als zusätzliche Sprache bezeichnet wird". Das Dokument hebt auch die Tatsache hervor, dass die Artikel 12, 26 und 31 des LDB eine wichtige Unterstützung für das Projekt darstellen, da sie die Möglichkeit einer diversifizierten und flexiblen Strukturierung der Schulen zulassen und die Autonomie ihrer Organisation für den kollektiven Aufbau jedes pädagogischen Projekts anerkennen.

Bei den deutschsprachigen Schulen bestanden von Anfang an Partnerschaften mit dem Goethe-Institut Rio de Janeiro und den öffentlichen Universitäten des Bundesstaates Rio de Janeiro (Universidade do Estado do Rio de Janeiro, Universidade Federal do Rio de Janeiro und Universidade Federal Fluminense), die Deutschlehrer*innen ausbilden. Eines der Hauptziele der gemeinsamen Bemühungen der Partner konzentrierte sich auf die Fortbildung von Lehrer*innen, die im Rahmen des Projekts den Unterricht mit Schwerpunkt Deutsch aufnehmen würden.

An dieser Stelle sei darauf hingewiesen, dass es zu jenem Zeitpunkt keine öffentliche Ausschreibung für die Stelle eines Deutschlehrers/ einer Deutschlehrerin gab. Das städtische Schulamt der Stadt Rio de Janeiro (SME - Secretaria Municipal de Educação) suchte unter den verbeamteten Lehrer*innen nach denjenigen, die einen Abschluss im Sprachenpaar Deutsch-Portugiesisch besaßen. Ende 2017 bekundeten etwa 45 Lehrer*innen ihr Interesse an einer Teilnahme an dem Projekt, da sie Portugiesisch und Deutsch studiert hatten oder bereits eine persönliche Verbindung zur deutschen 
FERREIRA, M.V.; MARQUES-SCHÄFER, G.; BOLACIO FILHO, E. - DaF an öffentlichen Schulen

Sprache hatten. Aufgrund logistischer Probleme konnten jedoch nicht alle Interessenten aufgenommen werden. Infolgedessen startete das Projekt 2018 mit fünf Lehrer*innen, die dann an drei verschiedenen Schulen arbeiteten.

Die meisten Lehrer*innen dieser Gruppe waren vor mehr als zehn Jahren ausgebildet worden, was die Bedeutung ihrer Weiterbildung vor Beginn des Unterrichts in den für das Projekt ausgewählten Schulen deutlich erhöhte. Es ist wichtig zu erwähnen, dass die drei Schulen, die sich derzeit im Programm befinden, erst nach Befragungen in der Schulgemeinschaft und dann nach SME-Kriterien ausgewählt wurden (es gab 6 interessierte Schulen, 3 wurden für die zweisprachigen Schulen mit der deutschen Sprache ausgewählt). So haben die SME und das Goethe-Institut (GI) Rio de Janeiro zusammen mit der UERJ einen ersten Plan für diese Ausbildung ausgearbeitet: Das GI bot Stipendien für Sprachkurse und Fortbildungen für die am Projekt beteiligten Lehrer*innen an, und es wurde ein Programm mit Themen erstellt, die in Treffen und Workshops mit Unterstützung von Dozent*innen der Partneruniversitäten (derzeit die drei Universitäten, aus denen die Partnerschaft besteht) sowie von Privatschulen in Rio mit umfassender Erfahrung im Deutschunterricht für Kinder und Jugendliche der Grundschule (insbesondere die deutsche Corcovado-Schule und die schweizerischbrasilianische Schule) behandelt werden sollten. In diesen Fortbildungsveranstaltungen wurden u.a. folgende Themen diskutiert: Didaktik der Mehrsprachigkeit und Interkulturalität im Deutschunterricht für Kinder; Methodik und Analyse von Unterrichtsmaterialien für Kinder und Jugendliche; Lehrplan- und Aktivitätenplanung; Musik und Phonetik.

Die drei Schulen, in denen das Projekt der zweisprachigen deutschen Sprachschulen durchgeführt wird, liegen in peripheren Vierteln der Stadt Rio de Janeiro, und die Schulgemeinschaft setzt sich aus Familien zusammen, die meist nur über wenige oder begrenzte finanzielle Mittel verfügen.

Die Unterstützung der Arbeit der Lehrer*innen, die in den Klassenzimmern vor der Klasse stehen und nicht nur Deutsch, sondern auch Spanisch, Französisch und Englisch in den öffentlichen Schulen unterrichten, ist für uns von grundlegender Bedeutung, um die anvisierten Ziele zu erreichen. Diese Unterstützung kann nicht nur materieller Art sein, damit ihre Arbeit von Erfolg gekrönt ist, sondern auch durch Partnerschaften mit ausländischen Einrichtungen (in diesem Fall dem Goethe-Institut) und öffentlichen Universitäten, die zukünftige Sprachlehrer*innen ausbilden. Der 
FERREIRA, M.V.; MARQUES-SCHÄFER, G.; BOLACIO FILHO, E. - DaF an öffentlichen Schulen

Lerneffekt aus dieser Synergie zwischen Theorie und Praxis war sehr groß und extrem wichtig für die Ausbilder*innen, um auf dem Laufenden zu bleiben und immer Kontakt zur Praxis zu haben. Vor allem wenn man berücksichtigt, dass in der Ausbildung von Fremdsprachenlehrer*innen - insbesondere Deutsch - in der Regel die ersten 4 Jahre der Grundschule nicht thematisiert werden, da man ja in Brasilien in der Regel Fremdsprachen erst nach dem 5. Schuljahr lernen kann. Dies ändert sich jedoch seit einigen Jahren durch die Aktionen der drei im beschriebenen Projekt vertretenen Universitäten, die sich immer mehr in ihren jeweiligen Didaktik- und Methodikseminaren dem Primarbereich widmen.

Die Hauptherausforderungen des Projekts lassen sich in zwei Hauptachsen auflisten: 1) kontinuierliche, auf den Kontext der Unterrichtspraxis ausgerichtete Lehrerausbildung und 2) die Ausarbeitung von Lehrplänen und Lehrmaterial.

Unter dem Aspekt der Weiterbildung kann der Vorschlag, Communities of Practice (ECKERT 2000) zu bilden, für die Verbesserung des Lehrwissens vielversprechend sein. Die Stärkung der Identität des Pädagogen als Erforscher seines eigenen Unterrichts, der Anreiz, ein Werk mit kollektiven Reflexionen und abgestimmt auf die Anforderungen der Schulgemeinschaft aufzubauen, in dem der Unterricht zunehmend auf die Inhalte und das transversale Wissen der Grundbildung ausgerichtet werden muss, tragen zur Vision des Lehrers als Sprachpädagoge bei, d.h. seine soziale Rolle besteht nicht nur darin, Sprachen zu unterrichten, sondern durch Sprache zu unterrichten. Dieser Paradigmenwechsel, die Abkehr von der isolierten zu einer integrierten Arbeit, ist kein leichter Schritt. Aus diesem Grund müssen die pädagogische Leitung und das Koordinationsteam auf diesen Ansatz ausgerichtet werden, um Ausbildungsräume innerhalb der Schule selbst zu fördern. Der Austausch bewährter Praktiken bei Treffen, die Erstellung des Unterrichtsplans gemeinsam oder mit PeerReview, ja sogar durch zwei Klassenlehrer*innen, in kurzen oder experimentellen Kontexten, können die wichtigsten Maßnahmen sein, die auf die lang erträumte Integration zwischen der Planung der Lehrer*innen von Zusatzsprachen und des Generalisten oder Fachlehrers im Kontext der interkulturellen und mehrsprachigen Bildung hindeuten. Hinzu kommt der Vorschlag, den Austausch zwischen Schulen zu fördern, wenn möglich durch Seminare oder Konferenzen.

Im Bereich der deutschsprachigen Lehrplan- und Materialentwicklung kann der Vorschlag der Weiterbildung dazu beitragen, Fähigkeiten und Fertigkeiten abzubilden, 
FERREIRA, M.V.; MARQUES-SCHÄFER, G.; BOLACIO FILHO, E. - DaF an öffentlichen Schulen

die den Erwartungen der lokalen Realität entsprechen. Auf diese Weise erfolgt die Umsetzung des Curriculums von der Mikroebene auf die Makroebene, d.h. von der Erfahrung des Schulraums auf die Institution SME. Für das Projekt wurde daher kein Lehrplan im Voraus erstellt, der als Leitbild angenommen werden sollte, sondern was es heute im Rahmen des Projekts gilt, spiegelt das wider, was Lehrer*innen und Schulgemeinschaft von Deutschlernen erwarten. In diesem Sinne wird die Arbeit bis jetzt auf der Grundlage der Planung der Lehrkräfte durchgeführt. Die Ziele für jedes Schuljahr werden durch eine Kooperation zwischen Lehrkräften, Schulleitung, Koordinatoren der Bildungsabteilung der SME und Universitätsprofessoren formuliert.

\subsection{Der Fokus auf Literacy-Praktiken und die interkulturelle Kompetenz der Lernenden}

\subsubsection{Literacy als soziale Praxis}

In den letzten Jahrzehnten beziehen sich einige Kernkonzepte, die die Prozesse des Lehrens und Lernens von Fremdsprache insbesondere in öffentlichen Schulen im brasilianischen Kontext geleitet haben, auf die Idee von Literacy als soziale Praxis (literacy practices). Begriffe wie kritische Literacy, neue Literacys und auch Multiliteracies werden häufig in der Diskussion verwendet. Knapp (2014: 127) plädiert dafür, dass in Bezug auf den Wissenserwerb in der Schule der Begriff "akademische Literacy" verwendet wird, da Literacy als die Fähigkeit verstanden wird, die Schriftsprache im jeweiligen soziokulturellen Kontext zu verwenden (KNAPP 2014: 128). Nach diesen Annahmen bedeutet Literacy-Fähigkeiten zu entwickeln, demnach nicht nur Lesen und Schreiben zu lernen, sondern auch die Fähigkeit auszubilden, sich am literalen Diskurs einer Gesellschaft im jeweiligen soziokulturellen Kontext $\mathrm{zu}$ beteiligen (SCHMÖLZER; EIBINGER 2008: 40).

Ein weiterer wichtiger Aspekt bei der Erörterung des Konzepts der Literacy ist, dass seine Definitionen oft im Gegensatz zum Begriff der Alphabetisierung geprägt ist. Soares (2009: 21) erklärt, dass Alphabetisierung eine Konstruktion ist, die traditionell mit mechanischen Prozessen der Kodifizierung und Dekodierung für den Akt des Lesens verbunden war. Andererseits extrapoliert der Begriff der Literacy diese mechanistische Vision des Lesens und bezieht in den Akt des Lesens das Engagement des Individuums für die verschiedenen sozialen Praktiken ein, die den Lese- und Schreibprozess in den verschiedensten Kontexten betreffen, wie z.B. die visuelle Literacy oder die digitale Literacy (d.h. die Art und Weise des Lesens und des Umgangs mit Bildern und digitalen 
Ressourcen). Knapp (2014) fügt hinzu, dass Studien zur Literacy-Praxis auf den konkreten Praktiken des Menschen mit Text und auf diesem Wissen für ein besseres Verständnis des sozialen und kulturellen Umfelds basieren.

Im deutschsprachigen Kontext wird oft auf die Verbindung zwischen den Begriffen Literacy und Textkompetenz hingewiesen. Nach Schmölzer und Eibinger (2008: 42) bezeichnet der Begriff der Textkompetenz, die Fähigkeit Texte zu lesen und zu verstehen, um handeln zu können. Es ist jedoch wichtig, die kritische Komponente hervorzuheben, die mit der Idee der Literacy verbunden ist, die zu einem großen Teil auf die Inspiration durch die Arbeit von Freire zurückgeht (1979; 1999; 2001; 2002; 2005). In diesem Sinne ist die Literacy mit der Mobilisierung des Vorwissens der Lernenden verbunden, die durch den Kontakt mit neuem Wissen und neuen Perspektiven zu Agenten werden, die in der Lage sind, sich in der Gesellschaft zu engagieren. Dieser Annahme folgend bezieht sich für Mattos (2011) die Literacy auch darauf, wie der Lernende sich in Praktiken engagiert, die die Sprache in der Welt einbeziehen, indem er effektiv und kritisch handelt und somit zur Entwicklung der aktiven Teilhabe als Bürger*in in der Gesellschaft und zur Bildung von Agenten der Transformation der Gesellschaft beiträgt. Nach Ansicht der Autorin waren es gerade diese Veränderungen, die unsere Welt durchläuft, die den Bedarf an neuen Generationen von Lehrlingen zum Erwerb neuer Fertigkeiten mit sich brachten und so das Konzept der „neuen Literacys“ hervorbrachten. Auf diese Weise wird sie zu einer wichtigen Rolle der Schule, neben der Bildung von Individuen mit vielfältigen Kenntnissen, die Bildung von Bürgern. Der Begriff Bürger wird hier wie bei Ruscheinsky (1999: 30) verstanden: derjenige, der die Fähigkeit hat, an Entscheidungen, die sein Leben direkt betreffen, teilzunehmen und sich in sie einzumischen, von seinem Zugang ,zu der Vielzahl von Räumen, in denen er dieses Recht ausüben kann“. Noch immer plädieren die Autoren Menezes de Souza und Monte Mór (2006: 91) für die Verbindung zwischen kritischer Literacy und aktiver Teilhabe in der Gesellschaft:

Bürger zu sein bedeutet, zu verstehen, welche Position/Platz eine Person (der Student, der Bürger) in der Gesellschaft einnimmt, d.h. aus welcher Sprechposition sie in der Gesellschaft spricht. Warum ist dies ihre Position? Wie ist es dorthin gekommen? Möchte er dabei sein? Möchte er es ändern? Möchte er da rauskommen? Schließt diese Position Sie ein oder schließt Sie wovon aus?

Neben den Konzepten der kritischen Literacy und der neuen Literacys gibt es auch das Konzept der Multiliteracy. Für Rojo (2012) umfasst und transponiert Multiliteracy 
FERREIRA, M.V.; MARQUES-SCHÄFER, G.; BOLACIO FILHO, E. - DaF an öffentlichen Schulen

die beiden anderen, da es neben der Schrift auch die Artikulation von anderen Modalitäten wie Bild, Sprache und Musik umfasst.

Verschiedene Studien, wie z.B. Santos \& Ifa (2013) und Edmundo (2010) zeigen, dass der Fremdsprachenunterricht mit Schwerpunkt auf kritischer Literacy einen bedeutenden Einfluss auf die Erweiterung der Interpretationsfähigkeit der Schüler*innen und die Entwicklung ihres Bewusstseins für die verschiedenen Handlungsweisen in der Gesellschaft hat. Santos \& Ifa (2013), die den kritischen Unterricht im Englisch als Fremdsprache an öffentlichen Schulen untersuchen, weisen auf die zunehmende Wahrnehmung der Schüler*innen hin, dass Lernen etwas ist, das gemeinsam geteilt und kollektiv aufgebaut wird, und kommen so $\mathrm{zu}$ dem Schluss, dass Wissen etwas Aufgebautes ist, was zur Folge hat, dass es transformiert werden kann. Auch die Studie von Edmundo (2010) zeigt, dass in Praktiken des Fremdsprachenunterrichts aus der Perspektive der kritischen Literacy die Fähigkeit der Schüler*innen hervorgehoben wird, ihr Dasein in der Welt zu reflektieren. Nach Ansicht des Autors können die Aktivitäten, die in diesen Klassen durchgeführt werden, zur Förderung von Individuen beitragen, die in der Lage sind, zu handeln und ihre Realität zu verändern, und folglich die Gesellschaft als Ganzes zu transformieren.

Menezes de Souza und Monte Mór (2006) plädieren dafür, dass der Kontakt der Lernenden mit einer neuen Sprache/Kultur zu ihrer Anerkennung als Subjekte beitragen soll, indem sie über die gemeinsamen Werte in ihrer Gemeinschaft nachdenken. Auf diese Weise werden sie ermutigt, sich mit den Werten und Modellen anderer Kulturen auseinanderzusetzen, was zur Entwicklung eines kritischen Bewusstseins für die soziokulturelle und sprachliche Vielfalt beigetragen hat. Diese Reflexionen heben daher die Beziehung zwischen Fremdsprachenunterricht, kritischer Literacy und Interkulturalität hervor, wobei letztere das Konzept ist, mit dem wir uns im nächsten Abschnitt befassen werden.

\subsubsection{Zur interkulturellen kommunikativen Kompetenz}

Die Diskussion um interkulturelles Lernen ist ein festes Thema im Bereich der Fremdsprachendidaktik. Im Zuge einer stärkeren Betonung des pädagogischen Auftrags des Fremdsprachenunterrichts werden als Ziele des Fremdsprachenunterrichts nicht mehr nur die Vermittlung von Sprachkenntnissen, sondern auch die Begegnung verschiedener Kulturen und die Reflexion darüber. Dazu gehört unter anderem die konsequente 
FERREIRA, M.V.; MARQUES-SCHÄFER, G.; BOLACIO FILHO, E. - DaF an öffentlichen Schulen

Einbeziehung der Fremdperspektive in den Lernprozess. Diente die Landeskunde im Fremdsprachenunterricht zuvor überwiegend der Vermittlung von Informationen über das Zielsprachenland, so wurde sie nun in den Dienst der Völkerverständigung gestellt. Sie sollte nicht mehr nur enzyklopädisches Wissen vermitteln, sondern eine Reflexion über die eigene und die fremde Kultur ermöglichen. Ziele dieser Neubestimmung der Landeskunde waren die Sensibilisierung der Lernenden für Unterschiede zwischen den Kulturen, der Abbau von Vorurteilen und Klischees, sowie die Förderung von Toleranz und einer interkulturellen kommunikativen Kompetenz (IKK).

Um diese Ziele zu erreichen, wurden in verschiedenen Ländern unterschiedliche Methoden und Konzepte entwickelt, die mit empirischen Studien in der Praxis ausprobiert wurden. Bis heute ist das Werk von Byram (1997) "Model for Intercultural Comunicative Competence" einer der einflussreichste Beitrag zu dieser Diskussion. In seinem Buch beschreibt der Autor fünf Dimensionen für den Erfolg der interkulturellen Kommunikation und dabei wird deutlich, dass interkulturelle kommunikative Kompetenz kognitive, affektive, ethnische und Verhaltensstufen beinhaltet, die miteinander verbunden sind. Solche Aspekte sind auch mit kommunikativen, soziolinguistischen und diskursiven Kompetenzen in einem einzigen Umfeld verbunden, um die IKK zu erreichen.

Kinder im Grundschulalter verfügen aufgrund ihrer lern- und entwicklungspsychologischen Voraussetzungen nur teilweise über solche Kompetenzen. Byram (ebd.: 72) erkennt die Unterschiede zwischen Kindern und Erwachsenen und bezieht sich in seinem Modell lediglich auf die letzten. Brunsmeier (2016: 142) führt aus, dass es sehr spekulativ zu vermuten wäre, zu beschreiben, was Kinder in diesem Alter erreicht haben müssen, um IKK zu erreichen. Für die Autorin soll zunächst die Bedeutung von IKK sowie die Aufgaben zur Förderung von IKK für die Arbeit in der Grundschule adaptiert werden. Genau das versucht Brunsmeier in ihrer Studie zu realisieren, die sie im Englischunterricht in Deutschland durchführte.

So weist Brumsmeier gerade im Hinblick auf die Entwicklung der interkulturellen kommunikativen Kompetenz im Kindesalter darauf hin, wie wichtig es für Kinder ist, die Fremdsprache als eine Konstruktion zu erkennen, die in der Lage ist, "kulturelle Inhalte zu transportieren", d.h. es ist wichtig, dass sie wahrnehmen können, dass die Sprache ihnen den Kontakt mit anderen Menschen und anderen Kulturen ermöglicht und dass dieser Kontakt es ihnen erlaubt, Gemeinsamkeiten und kulturelle Unterschiede mit diesen 
FERREIRA, M.V.; MARQUES-SCHÄFER, G.; BOLACIO FILHO, E. - DaF an öffentlichen Schulen

anderen Menschen und Kulturen zu entdecken (BRUNSMEIER 2016: 37). Die Autorin fügt hinzu, dass ein wichtiges Ziel des Fremdsprachenunterrichts ist, Kinder neugierig zu machen auf das, was anders ist, und eine Offenheit für neue Sprachen und Kulturen zu fördern.

Die Entwicklung interkultureller Fähigkeiten bei uns selbst und bei unseren Lernenden setzt eine dialektische Vision der Vielfalt der Kulturen voraus, die es uns ermöglicht, Grenzen zwischen verschiedenen kulturellen Systemen zu überschreiten (AgUiAR 2010: 295) und die Unterschiede zwischen unseren Kontexten und Sozialpraktiken deutlich zu machen. Die Entwicklung interkultureller Kompetenzen bei uns selbst und bei unseren Lernenden setzt eine dialektische Vision der Vielfalt der Kulturen voraus, die es uns ermöglicht, Grenzen zwischen verschiedenen kulturellen Systemen zu überschreiten (ibid.: 295) und die Unterschiede zwischen unseren Kontexten und sozialen Praktiken deutlich zu machen. Die Forscherin Aguiar (2010) argumentiert daher, dass interkulturelle Kompetenzen Fähigkeiten zur Aufrechterhaltung der Kommunikation mit einem anderen Menschen sind, die aus anderen Referenzsystemen als den unseren stammen. Diese Art von Kompetenz ermöglicht es uns, einen Blick darauf $\mathrm{zu}$ werfen, wie Texte und Kontexte in verschiedenen sozialen Gruppen auf unterschiedliche Weise dialektisch interagieren, so dass wir die Tendenz eindämmen können, dem Andersartigen zu widerstehen oder sich kritiklos dem Fremden anzuschließen (AgUiAR 2010: 295). Auf diese Weise markiert die Autorin auch den Unterschied zwischen den Begriffen Multikulturalismus und Interkulturalität und weist darauf hin, dass der erste Begriff mit dem verbunden ist, was sie kulturelle Konvergenz nennt, während der Begriff interkulturell auf Dialog und kulturelle Interaktion anspielt und eine effektive Öffnung zum Anderen darstellt (AGUIAR 2010: 114).

Der gemeinsame europäische Rahmen (2000) stellt neben mehreren länderspezifischen Dokumenten die IKK als Kernkompetenz des Fremdsprachenunterrichts heraus. In diesen Dokumenten erscheint IKK mit dem gleichen Wert wie Sprachkenntnisse. Im brasilianischen Kontext stellt das Dokument des BNCCs die interkulturelle Dimension zusammen mit vier anderen Fähigkeiten (Mündlichkeit, Lesen, Schreiben und Sprachkenntnisse) als eine der grundlegenden Achsen für die Sprachausbildung dar. In dem Dokument wird die interkulturelle Dimension mit der Reflexion über Aspekte der Interaktion zwischen der Kultur der Schüler*innen und der Kultur englischsprachiger Menschen verknüpft, um die Koexistenz, den Respekt, die 
Überwindung von Konflikten und die Wertschätzung der Vielfalt zwischen den Völkern zu fördern (BRASIL 2017). Das BNCC stellt auch die Verwendung des Begriffs „Fremd“ in Frage und argumentiert, dass

in diesem Vorschlag die englische Sprache nicht mehr die des „Ausländers“ ist, der aus hegemonialen Ländern kommt und dessen Sprecher als Vorbild dienen, noch ist es eine Variante der englischen Sprache. In dieser Perspektive wird die Verwendung durch Sprecher, die über die ganze Welt verteilt sind und über ein unterschiedliches sprachliches und kulturelles Repertoire verfügen, begrüßt und legitimiert, was es zum Beispiel ermöglicht, die Auffassung in Frage zu stellen, dass das einzige ,richtige“ Englisch das von Amerikanern oder Briten gesprochene Englisch ist. (BRASIL 2017: 241)

Im Text des Amtsblatts der EMS (2018) finden sich auch Anmerkungen zur interkulturellen Erziehung, wie im folgenden Abschnitt (RIO DE JANEIRO 2018: 44):

Aus dem oben Gesagten geht hervor, dass in der zweisprachigen Schule das Lernen über die reine Beherrschung einer weiteren Sprache hinausgeht. Zweisprachigkeit erfordert mehr als den Erwerb von Sprache und sollte neben dem Kontakt mit einer anderen Sprache auch andere Erfahrungen und andere Sichtweisen auf die Welt ermöglichen.

Gemäß den staatlichen curricularen Richtlinien für die Grundbildung von Modernen Fremdsprachen, im Original Diretrizes Curriculares Estaduais - Línguas Estrangeiras Modernas (DCE-LEM) (PARANÁ 2008: 56): "Gegenwärtige Gesellschaften überleben nicht isoliert; sie stehen in Beziehung zueinander, überschreiten geopolitische und kulturelle Grenzen, kommunizieren und versuchen, einander zu verstehen". Laut den DCE-LEM (PARANÁ 2008: 56) "überleben heutige Gesellschaften nicht isoliert; sie stehen in Beziehung zueinander, überschreiten geopolitische und kulturelle Grenzen, kommunizieren und versuchen, einander zu verstehen". In demselben Dokument (2008: 56) wird in Bezug auf zusätzlichen Sprachunterricht folgendes Argument vorgebracht: "Sie sollte die Beziehungen berücksichtigen, die zwischen der untersuchten Sprache und der sozialen Eingliederung hergestellt werden können, mit dem Ziel der Entwicklung eines Bewusstseins für die Rolle der Sprachen in der Gesellschaft und der Anerkennung der kulturellen Vielfalt".

\section{Zu der Studie}

Die vorliegende Studie hat ein qualitatives Design interpretativer Natur (DENZIN; LinColn 2000, ChizzotTi 2006). Nach Moita Lopes (1994) ist der interpretative Charakter des wissenschaftlichen Schaffens der Geisteswissenschaften mit dem Zustand der Sprache selbst verbunden, die nach Ansicht des Autors sowohl die Ursache als auch die Lösung für die Interpretationsprobleme der Welt ist. In seinen Worten:

Pandaemonium, São Paulo, v. 25, n. 45, jan.-abr. 2022, p. 1-28 
FERREIRA, M.V.; MARQUES-SCHÄFER, G.; BOLACIO FILHO, E. - DaF an öffentlichen Schulen

Das Spezifische an der sozialen Welt ist die Tatsache, dass die Bedeutungen, die sie charakterisieren, vom Menschen konstruiert werden, der die Welt um sich herum interpretiert und uminterpretiert und so dort nicht eine einzige Realität, sondern mehrere Realitäten entstehen lässt (MOITA LOPES 1994: 331).

Der Korpus wird im Licht der Theorie der Inhaltsanalyse ausgewertet. Nach Olabuenaga und Ispizúa (1989) ist die Inhaltsanalyse eine Technik zum Lesen und Interpretieren des Inhalts verschiedenster Dokumente, die wichtige Erkenntnisse über Aspekte und Phänomene des sozialen Lebens liefern kann. Die inhaltliche Analyse in ihren qualitativen und interpretativen Aspekten ist Teil einer Reihe von Annahmen, die bei der Untersuchung eines Textes als Stütze zur Erfassung seiner symbolischen Bedeutung dienen. In diesem Verfahren werden die Kategorien, die in mündlichen oder schriftlichen Diskursen auftauchen, in Einheiten kodifiziert, die die Beschreibung und Analyse der relevantesten Wiederholungen und Merkmale der dort vorhandenen Inhalte ermöglichen. Nach Chizzotti (2006) ist diese Technik eine Möglichkeit, „[...] die Daten $\mathrm{zu}$ interpretieren und aus ihnen die Bedeutungen durch die elementaren Einheiten zu extrahieren, aus denen dokumentarische Produkte bestehen: Schlüsselwörter, Lexikon, spezifische Begriffe, Kategorie und Themen [...]“ (CHIZzOTTI 2006: 115).

Hauptziel der vorliegenden Studie ist es, die Beiträge des Unterrichts Deutsch als Fremdsprache im zweisprachigen Schulprojekt der Stadt Rio de Janeiro zur Entwicklung interkultureller Kompetenz von Schüler*innen zu untersuchen, die nicht nur auf der Arbeit mit kulturellen Elementen im Unterricht, sondern auch auf der Betonung der in den Aktivitäten durchgeführten Unterrichtspraktiken basieren.

Um die Perspektiven der Lehrer*innen, die in den an der Studie beteiligten Schulen ihre pädagogische Arbeit mit der deutschen Sprache entwickelt haben, zu erfassen, wurde ein online Fragebogen entwickelt, dessen Link den Lehrer*innen per EMail geschickt wurde.

Die Forschungsteilnehmer sind also die Lehrer*innen, die sich am Projekt „Deutsch in den öffentlichen Schulen Rios“ beteiligt haben. Obwohl sie als Deutsch- und Portugiesischlehrer*innen ausgebildet sind, arbeiteten sie in den letzten ihres Berufslebens nur mit Portugiesisch, da Deutsch kein Schulfach im Bundesland Rio de Janeiro ist. Relevant ist auch die Tatsache, dass alle Lehrer*innen über langjährige Lehrerfahrung im öffentlichen Dienst verfügen. Ihre Erfahrungen als Deutschlehrer*innen begannen jedoch, mit Ausnahme einer Lehrerin, mit der Einführung des Projekts der zweisprachigen Schulen der Stadt Rio. 
FERREIRA, M.V.; MARQUES-SCHÄFER, G.; BOLACIO FILHO, E. - DaF an öffentlichen Schulen

Für die vorliegende Studie wurde ein Fragebogen entwickelt, der aus neun offenen Fragen besteht (siehe Anhang). Diese Fragen zielten darauf ab, neben einigen allgemeineren Informationen, wie Ausbildung und Erfahrung zu sammeln, vor allem zu eruieren, wie die Lehrer*innen das Projekt bewerten, was sie dazu bewogen hatte, mitzumachen und wie sie den Beitrag des Projekts bzw. des Deutschunterrichts zur Entwicklung von interkultureller Kompetenz und kritischer Literacy einschätzen.

\section{Annäherung an die Daten}

Der für unsere Studie eingesetzte Fragebogen wurde den sechs Lehrer*innen, die in den drei Schulen, die für den Deutschunterricht am bilingualen Projekt der Stadt Rio de Janeiro zuständig sind, über ein online Formular zur Verfügung gestellt. Alle Lehrer*innen beantworteten die dort gestellten Fragen. Wir werden dann mit der Analyse der Antworten der Lehrer*innen fortfahren und dabei vor allem den zentralen Schwerpunkt unserer Studie erörtern: die Literacy-Praktiken (práticas de letramento), die mit den Aktivitäten und Lehrmethoden verbunden sind, und die Entwicklung der interkulturellen Kompetenz der Schüler*innen.

Die ersten beiden Fragen des Fragebogens betrafen Daten über das Profil der Lehrkräfte. In diesem Zusammenhang ist es wichtig hervorzuheben, dass vier der sechs Lehrer*innen, die an der Umfrage teilgenommen haben, angaben, dass sie seit mehr als zehn Jahren an den öffentlichen städtischen Schulen von Rio de Janeiro arbeiten, während zwei der derzeitigen Lehrer*innen zwischen fünf und zehn Jahre Erfahrung in diesem Kontext haben. Das ist eine Tatsache, die von der großen Erfahrung dieser Lehrende im Kontext der öffentlichen Schule zeugt. Von allen sechs Lehrkräften gab nur eine an, dass sie vor dem Projekt Deutsch unterrichtet hatte. Mit anderen Worten, obwohl sie den Dopppelstudiengang Deutsch-Portugiesisch abgeschlossen hatten, begannen alle anderen Lehrer*innen erst nach Beginn des Bilingualen Schule-Projekts Deutsch zu unterrichten. Dies ist sehr bemerkenswert, denn die Lehrer*innen mussten einen Prozess der Weiterbildung bzw. „Umschulung“ durchlaufen, der sicherlich nicht nur einen starken Einfluss darauf hatte, was sie für ihre Klassen zu entwickeln begannen, sondern auch auf ihr eigenes Verständnis dieser Praktiken.

Bezüglich der Frage 3 des Fragebogens, die sich mit den sprachlichen Themen befasst, die sie in ihren Klassen bearbeitet haben, ist es interessant festzustellen, dass sich 
die aufgeführten Themen nicht wesentlich von den Aspekten unterscheiden, die normalerweise auch in regulären Kursen für Deutsch als Fremdsprache behandelt werden, wenn man die Altersgruppe der Lernenden berücksichtigt:

P19 : „Meine Schüler waren im Durchschnitt 7, 8 Jahre alt. Im Hinblick auf diese Altersgruppe habe ich den Unterricht auf die Entwicklung [folgender] Sprachkenntnisse ausgerichtet: sich vorstellen, wo er/sie wohnt, sein/ihr Alter und auch Fragen zum Thema stellen; Zahlen bis zehn und Vorkenntnisse von Zahlen bis 20; Farben; Freizeit- und Sportaktivitäten; das Klima auf verschiedene Weise beschreiben; über Familienmitglieder sprechen; Tiere beschreiben (Bremer Musiker); Kommandos auf Deutsch verstehen und auch reproduzieren." ${ }^{\text {10 }}$

Auf die Frage nach den Zielen, die sie als die wichtigsten für den Deutschunterricht in der Schule wählten, gingen die an den häufigsten markierten Aspekten jedoch weit über die sprachlichen hinaus. Wenn es also um streng sprachliche Ziele ging, wurde nur die Betonung der Entwicklung der Mündlichkeit (von zwei Lehrern erwähnt) erwähnt:

P1: „Die Entwicklung des mündlichen Ausdrucks in deutscher Sprache.“

P4: „Priorisierung der Basiskommunikation in deutscher Sprache.“

Andererseits war der meistgenannte Aspekt in den Antworten der Lehrer*innen die Einfügung kultureller Elemente in die Unterrichtspraxis, worauf fünf der sechs Befragten hinweisen:

P1: „Einführung des Schülers in den kulturellen Kontext der deutschsprachigen Länder (Sport, Tänze, Feste, große Schriftsteller, Traditionen von Festterminen...); [...] Annäherung an die jeweilige Literatur."

P2: „Aneignung nicht nur der Sprache, sondern auch der Interaktion des Schülers mit der Kultur."

Ein weiterer Aspekt, an den sich zwei Lehrer erinnerten, war die Bedeutung der Erweiterung der Weltsicht der Schüler und die Entwicklung einer offenen Haltung für neues Wissen bei den Schüler*innen:

P3: „Es ist eine Möglichkeit, den kulturellen Horizont der Lernenden zu erweitern, ihr Leben mit neuem Wissen über die Welt zu bereichern und ein Fenster der Möglichkeiten für zukünftige berufliche Möglichkeiten zu öffnen.“

P4: ,„...] das Wissen der Schüler über die Welt zu erweitern.“

Als nächstes nennen wir weitere Punkte, die ebenfalls von allen Lehrenden erwähnt werden: emotionale Aspekte (wie Freude am Lernen und Motivation), die Erweiterung der beruflichen Möglichkeiten und die Anerkennung der eigenen Identität

\footnotetext{
${ }^{9}$ P steht hier für "professor" oder "professora", auf Deutsch Lehrer bzw. Lehrerin.

${ }^{10}$ Alle in diesem Kapitel zitierten Aussagen der Forschungsteilnehmer*innen wurden von den Autor*innen ins Deutsche übersetzt.
} 
durch die Schüler*innen sowie von Identitäten, die sich von der eigenen unterscheiden. Die folgenden Auszüge veranschaulichen diese Aspekte:

P2: „den Zugang zu neuen Methoden zu erweitern und die Schulerfahrung zu einer angenehmen Erfahrung umwandeln.“

P3: „[das Deutschlernen bedeutet] ein Möglichkeitsfenster für zukünftige berufliche Möglichkeiten.“

P6: ,[...] Ich verstehe, dass über den sprachlichen und kulturellen Aspekt hinaus die Frage nach der eigenen Identität und dem Verständnis und der Akzeptanz verschiedener Kulturen ein sehr interessanter Schritt in der Ausbildung von Kindern und Jugendlichen ist. Es geht nicht nur um den grammatikalischen Inhalt einer anderen Sprache. Es geht um die Konstruktion der Individualität jedes einzelnen am Prozess Beteiligten.“

Die nächste Frage (Frage 4) befasste sich konkreter mit den kulturellen Aspekten, die von den Lehrenden für die Unterrichtsaktivitäten mit den Schüler*innen gewählt wurden. In den Antworten konnten nicht nur einige Themen festgestellt werden, die häufiger bearbeitet wurden, sondern wir konnten auch einige Ansätze und Methoden beobachten, die verwendet wurden. In Bezug auf die Themen wurde oft die Arbeit mit Märchen und Kurzgeschichten (hauptsächlich das Märchen „Bremer Stadtmusikanten“ erwähnt, das als Miniprojekt mit den Schüler*innen von zwei Schulen verwendet wurde) zu finden.

Ein weiteres Thema, das in den Antworten häufig erwähnt wurde, war die Arbeit mit Gedenktagen (Weihnachten, Ostern, Muttertag u.a.), und in Bezug auf diesen Punkt nennen einige Lehrer*innen als Hauptziel den Kulturvergleich und die Herstellung von Beziehungen zwischen den verschiedenen kulturellen Praktiken. In den folgenden Ausführungen können wir diese Argumente beobachten:

P5: „Karneval, Ostern, Muttertag, [...] Kindertag, Weihnachten. Denn diese Feste waren Teil des Schulkalenders. Ich versuchte, mich den Themen anzunähern, indem ich die Feierlichkeiten in beiden Kulturen präsentierte.“

Ein weiteres Thema, das in den Antworten der Lehrer*innen sehr präsent ist, ist die Verbindung der Arbeit mit kulturellen Themen zu Schulveranstaltungen oder kleinen kulturellen Darbietungen wie Tänzen, kleinen Theaterstücken oder Chören für die Schulgemeinschaft (andere Klassen und Eltern und Erziehungsberechtigte):

P1: „Veranstaltung Brasilien-Deutschland, Party mit Tanzvorführungen, Theater, typischem Essen, typischen Spielen, Plakaten und Ständen mit deutscher Dekoration. [...] Unser Mini-Oktoberfest, mit Plakaten, Präsentation von Arbeiten, Modelle, von Schüler*innen gemachte Objekte, Videopräsentation (Kino), Tanz, Theater, auch für die Gemeinschaft. Abschlusspräsentation in den zweisprachigen Englisch-Sprachschulen.“

In Bezug auf die Methodik der Arbeit und der durchgeführten Aktivitäten kann man die Betonung der Interdisziplinarität feststellen, da in den Aussagen einiger 
Lehrkräfte die Arbeit mit Themen aus dem Bereich Geografie, Künste, Theater und Musik genannt wird, wie es in der Antwort von P1 beschrieben wird:

P1: „[Sie wurden aus der Erzählung Bremer Stadtmusikanten entnommen] Geographische, soziokulturelle Aspekte, lexikalische Arbeit, Theateraufführung, Tanzdarstellung, Kunstwerke (Anfertigung der Szenerie der Stadt Bremen, Faltbuch, Kostüme...alles zusammen mit den Kindern) und Geschichtenerzählen.”

Es ist auch festzustellen, dass die Interaktion, die aktive Teilnahme der Schüler*innen im Mittelpunkt des Unterrichts steht, da einige Lehrer*innen auch von Aktivitäten wie die Präsentation von Texten durch Schülergruppen, dramatisierte Lesungen, für andere Gruppen oder Klassen berichten.

P1: "Andere Geschichten wurden intensiv bearbeitet und in ihren verschiedenen Aspekten untersucht, wie z.B. Schneewittchen, Rotkäppchen, und die Klassen hatten die Aufgabe, andere Klassen zu besuchen und sich gegenseitig die Geschichten zu erzählen, die für sie bestimmt wurden (mit Kostümen und dem Mischen von Sätzen auf Deutsch)".

Die Frage 5 des Fragebogens ergänzt die vorhergehende Frage, denn die Lehrer*innen werden darum gebeten, etwas mehr über die durchgeführten Aktivitäten zu erzählen (Frage 5: Wie wurden die Aktivitäten durchgeführt, bei denen diese kulturellen Aspekte angesprochen wurden? Bitte erzählen Sie uns ein wenig mehr darüber.). Auf diese Weise wurden die kulturellen Projekte und Veranstaltungen, die während des gesamten Schuljahres stattfanden, erneut erwähnt. Es ist wichtig hervorzuheben, dass einige Lehrer*innen das große Engagement der Schulgemeinschaft, einschließlich der Eltern und Erziehungsberechtigten der Schüler, betonten:

P1: „Die Schule „CIEP Professor Darcy Ribeiro“ (Campo Grande) begrüßte die Zweisprachigkeit in der Schule. Die Projekte zählten auf eine große Unterstützung durch die Institution und die Lehrer. Die Aktivitäten in der Klasse, die Veranstaltungen in der Schule, die Besuche, alles wurde organisiert, mit viel Engagement, einschließlich viel finanzieller Hilfe und Aufregung von Seiten der Eltern und Lehrer*innen.“

Neben der aktiven Teilnahme der Gemeinschaft an den Veranstaltungen und kulturellen Präsentationen der Schüler*innen erwähnten einige Lehrer*innen, dass diese Aktivitäten oft Aspekte wie das Gefühl der Zugehörigkeit zur Gruppe, den Respekt vor Diversität und die Entwicklung eines Bürgerbewusstseins förderten:

P6: „Das Projekt der Bremer Stadtmusikanten wurde aus der brasilianischen Adaption „Os Saltimbancos" bearbeitet. Die Hauptthemen waren Zusammenarbeit, Respekt für Unterschiede und Natur sowie der Wichtigkeit des Bürgerbewusstseins.“

Die Frage 6 bezieht sich speziell auf die Entwicklung der interkulturellen Kompetenz der Schüler*innen und lautete: ,Wie können diese Aktivitäten nach Ihrer Einschätzung zur Entwicklung der interkulturellen Kompetenz der beteiligten 
FERREIRA, M.V.; MARQUES-SCHÄFER, G.; BOLACIO FILHO, E. - DaF an öffentlichen Schulen

Schüler*innen beitragen?" Es ist interessant festzustellen, dass drei der fünf eingereichten Antworten (eine Lehrkraft hat die Frage nicht beantwortet) nicht direkt auf die Frage eingingen, nämlich wie die Aktivitäten tatsächlich zur Entwicklung der interkulturellen Kompetenz der Schüler*innen beigetragen haben. Eine der Antworten nannte den Beitrag zur Entwicklung der sozio-affektiven Fähigkeiten der Schüler*innen als Problemlösungskompetenz oder Steigerung des Interesses und der Motivation der Schüler*innen:

P2: „Sie tragen zu einer besseren Organisation im Klassenzimmer und zur Lösung von Problemen bei, die bei der Ausführung auftreten, Enthemmung, größere Aufmerksamkeit beim Spracherwerb.“

P4: „Diese Aktivitäten haben ein größeres Interesse der Schüler an der zu erlernenden Sprache gefördert, was die Arbeit des Lehrers sehr erleichtert hat.“

Nur zwei der Befragten präsentierten Beiträge, die sich auf Aspekte der interkulturellen Kompetenz beziehen können, wie z.B. die Wahrnehmung anderer Fächer und anderer Formen des Daseins in der Welt und die Entwicklung der Fähigkeit zur Akzeptanz und Toleranz gegenüber anderen Menschen und Kulturen, wie wir in den Antworten unten sehen können:

P1: „Der Beitrag ist so bereichernd, dass er schwer zu beschreiben ist! Die Schüler*innen haben eine neue Vorstellung von der Welt um sie herum kennen gelernt, ein einzigartiges Verständnis dafür, dass die Kenntnis einer Fremdsprache die Türen des Geistes öffnet und das Individuum neu erschafft. Die Kinder verstanden, dass die Welt über den kleinen Stadtteil, in dem sie leben, hinausgeht, sie erkannten, dass sie weit gehen können, sie wurden geografisch und emotional verortet und als Individuen umgestaltet, mit dem Ziel, Deutschland eines Tages kennen zu lernen.“

P3: „Sie trägt zur Akzeptanz und Toleranz dessen bei, was anders ist als wir.“

Die Fragen 7 und 8 bezogen sich auf die Auswirkungen des Deutschunterrichts für Schüler*innen in kognitiver, soziokultureller und emotionaler Hinsicht, die so formuliert wurden: Frage 7: „,Wenn Sie sich jetzt auf die Entwicklung von Kindern konzentrieren, wie können Sie aus Ihrer Erfahrung als Lehrer*in die Auswirkungen des Deutschunterrichts auf Ihre Schüler*innen in kognitiver/pädagogischer und soziokultureller Hinsicht beschreiben? “ Frage 8: ,und in emotionaler Hinsicht?““

Die affektiv-emotionalen Aspekte wurden in den Antworten der Lehrer*innen am häufigsten genannt. Es wurden nämlich Aspekte wie erhöhtes Selbstwertgefühl, Interesse und Motivation in den Antworten hervorgehoben:

P2: „Der Spracherwerb ging schnell, sie hatten das Gefühl, dass sie etwas verstehen konnten, was sie für schwierig hielten, und andere sagten, es sei unmöglich. In emotionaler Hinsicht hat der Einsatz von Spielen und Musik im Unterricht die Schüler*innen erfreut, die allmählich erkennen, dass sie mit mehr Freude lernen können.“ 
FERREIRA, M.V.; MARQUES-SCHÄFER, G.; BOLACIO FILHO, E. - DaF an öffentlichen Schulen

P6: „Das ganze Jahr über war das Feedback von anderen Lehrern und Familienmitgliedern, dass sich die Kinder in ihrer Disziplin, in ihrer Vorliebe für das Lernen und Sprechen der deutschen Sprache, in der Freude am Schulbesuch und an der Geselligkeit in der eigenen Familie verbessert haben.“

Außerdem hat der Deutschunterricht laut den Lehrern*innen stark zum Verständnis soziokultureller Aspekte beigetragen, d.h. zum besseren Verständnis bei den Schülern*innen ihres eigenen soziokulturellen Kontexts, sowie zum besseren Verständnis anderer Formen in der Welt zu leben und die Entwicklung von Respekt vor dem Andersartigen genannt wurden.

P1: „Das Verständnis, dass es verschiedene Gesellschaften und Kulturen gibt, ließ sie einander besser verstehen, entwickelte Respekt und Bewunderung und den Wunsch, Grenzen zu durchbrechen.“

P4: „Der Deutschunterricht hat ein großes Interesse an Sprache, Kultur und Geographie gefördert. Die Schülerinnen und Schüler konnten sich im geographischen Raum positionieren, indem sie verstanden, dass die Welt viel größer ist und Unterschiede zwischen den Völkern wahrnehmen. Es gab auch ein Interesse daran, in Deutschland und den Vereinigten Staaten zu studieren und zu arbeiten (sie lernen auch Englisch) und erkannten die Notwendigkeit, sich auf die Zukunft vorzubereiten.“

Als wichtigen kognitiven Gewinn für die Schüler*innen nannten die Lehrer*innen neben dem Erlernen der deutschen Sprache und der deutschen Kultur selbst das größere Verständnis der Schüler*innen für die Integration von Wissen und Fächern und die Entwicklung ihrer Fähigkeit, Beziehungen und Vergleiche zwischen ihnen herzustellen, vor allem sprachliche Beziehungen zwischen den Fächern, die auf Portugiesisch unterrichtet werden, der englischen Sprache (die auch in der Schule gelehrt wird) und der deutschen Sprache.

P1: „[...] die Entwicklung der Kinder war überraschend. Die öffentliche Schule hat viele bedürftige Kinder, die außerhalb der Schultore nicht gut essen, Hygiene nicht als wichtig erachten, in Häusern ohne gute physische oder psychische Struktur leben, nicht verantwortungsbewusst so präsent sind. In der Schule befinden sie sich in einer warmen Umgebung, ohne Klimaanlage, mit wenig Material. Trotzdem lernten sie, was sie auf Deutsch und über Deutschland, Österreich, die Schweiz konnten, trotzdem warteten sie gespannt auf den Moment des Unterrichts, sie wollten alles lernen, sich über alles erkundigen, im Unterricht nur Deutsch sprechen und hören, zeichnen, die Fahnen malen, die Lieder, Spiele und Witze lernen, die Videos anschauen, es war ein magischer Moment für sie.“

P2: „[Die Schüler*innen] brachten ihren Familien bei, was sie gelernt hatten, recherchierten im Internet über Deutschland und die deutsche Sprache und berichteten mit Spannung über die Ergebnisse ihrer Recherchen."

P3: „Der Kontakt mit einer anderen Sprache hat zu Vergleichen mit anderen Sprachen geführt, zu denen sie Zugang hatten, was sehr positiv ist.“

In der letzten Frage baten wir die Lehrer*innen, bis zu zehn Adjektive aufzulisten, die ihre Erfahrungen mit dem Deutschunterricht in ihren jeweiligen Schulen 
FERREIRA, M.V.; MARQUES-SCHÄFER, G.; BOLACIO FILHO, E. - DaF an öffentlichen Schulen

charakterisieren. Die Analyse der Antworten zeigt, dass von 37 zitieren Adjektiven 27 positiv und 10 negativ waren. Die häufigsten genannten positiven Adjektive waren spielerisch, lustvoll, lustig und aufregend. In Bezug auf die Schüler*innen und ihre Ausbildung nannten die Lehrkräfte die Adjektive kreativ, lohnend, bereichernd, anregend und ernsthaft. Die Adjektive mit einer negativen semantischen Belastung sind hauptsächlich mit den Schwierigkeiten verbunden, die im untersuchten Kontext auftreten. So wurden die Adjektive herausfordernd und erschöpfend zitiert. Dieses Ergebnis zeigt, dass die emotionale Bindung zum Projekt, der freie Raum für Kreativität und Unterrichtsgestaltung, den es in diesem Kontext gibt, für Engagement und Motivation seitens der Lehrkräfte sorgt.

Es lässt sich darüber hinaus aus den erhobenen Daten beobachten, dass Aspekte der Literacy-Praktiken und der Entwicklung der kommunikativen interkulturellen Kompetenz sehr betont auftauchten. Dies zeigt, dass sie für die Teilnehmer*innen der vorliegenden Studie sehr wertvoll sind. Dennoch waren die konkreteren genannten Beispiele, wie diese Praktiken gefördert werden, in einer zurückhaltenderen und reduzierten Form. Von den wenigen konkreten Beispielen, die in den Antworten der Lehrer*innen auftauchten, wurden vor allem Aktivitäten im Zusammenhang mit der Arbeit mit festlichen Tagen und Märchen genannt. In diesem Sinne könnten weitere Studien vertiefend diese Aktivitäten und ihren Beitrag für die Entwicklung der kommunikativen interkulturellen Kompetenz untersuchen.

Eine weitere Tatsache, die in der Studie auffällt, ist, dass gerade die Frage, die sich direkter damit befasste, wie die in den Klassen durchgeführten Aktivitäten zur Entwicklung der interkulturellen Kompetenz der Schüler*innen, nach Einschätzung der befragten Dozent*innen, beitragen könnten - nämlich die Frage 6 - sehr diffuse und ausweichende Antworten hatte. Daher legten viele der Antworten, die sich ergaben, ein größeres Gewicht auf sozio-emotionale Aspekte, wie z.B. die gesteigerte Fähigkeit der Schüler*innen, sich selbst zu organisieren und Konflikte in der Klasse zu lösen, und ihr gesteigertes Interesse an der deutschen Sprache. So können wir schlussfolgern, dass im Allgemeinen die sozio-emotionalen Aspekte bei der Analyse der Beobachtungen und Berichte der befragten Lehrer*innen in der gesamten Befragung im Vordergrund standen. Dementsprechend traten Aspekte wie Motivation, Interesse, Freude am Lernen sehr häufig in den Antworten auf und sind allem Anscheinen nach eine wichtige Grundlage für das Projekt. 


\section{Fazit}

Um die Fäden, die sich in den analysierten Daten ergeben haben, zu verknüpfen, ist es wichtig, die Ziele dieser Studie wieder zusammenzufassen, die darin bestanden, den Beitrag vom DaF-Unterricht im Rahmen zur kritischen Literacy und zur interkulturellen Kompetenz zu untersuchen und dabei die Enstehung des Projekts der zweisprachigen Schulen in Rio de Janeiro kurz darzustellen. In Anbetracht dieser Ziele und der Berichte der Lehrer*innen in unserer Forschung kann man behaupten, dass Initiativen wie das Projekt von grundlegender Bedeutung sind, damit Fortschritte in Richtung einer plurikulturellen und mehrsprachigen Ausbildung von immer mehr Schüler*innen ermöglicht werden können.

Anhand der mit den am Projekt tätigen Lehrenden durchgeführten Befragung konnte festgestellt werden, dass seine Nutzeffekte und Vorteile herausgehoben wurden, trotz der Herausforderungen, die allgemein mit dem Fremdsprachenunterricht in Brasilien verbunden sind, vor allem in Bezug auf die Sprachpolitik, zum Beispiel die Beschränkung auf Englisch als einzige Pflichtfremdsprache im BNCC - dem wichtigsten Dokument für die Grundbildung in Brasilien. Daher ist es möglich zu erkennen, dass die zitierten positiven Aspekte in der Analyse sehr betont erscheinen und dass die Motivation dieser Lehrer*innen ein sehr wichtiges Element in diesem Prozess ist.

In Bezug auf den durchgeführten Unterricht im Rahmen des Projektes stellen wir anhand der Äußerungen der Lehrenden fest, dass die in der Schule geförderten Praktiken, anstatt das Sprachwissen in den Vordergrund zu stellen, die Annahmen der Literacy und Interkulturalität als Leitfaden haben. Der Fokus liegt daher auf Aspekten der Kultur, der Entwicklung und der Erweiterung des Wissens der Schüler*innen, hauptsächlich durch Interdisziplinarität. In diesem Sinne ist es sehr relevant zu erwähnen, dass im Fragebogen als eines der Hauptziele des Deutschunterrichts und der von den Lehrer*innen erwähnten Aktivitäten, die Erweiterung des Weltwissens der Schüler*innen und ihre Offenheit für neues Wissen erscheint.

Auf jeden Fall ist zu beobachten, dass ein Großteil der von der Lehrkräfte beschriebenen praktischen Aktivitäten immer noch Gedenkdaten oder Märchen im Hintergrund haben, was an sich weitere Untersuchungen verdienen würde. Zwei weitere erwähnte Punkte, die wir für zukünftige Forschungen als gültig erachten, wären Aspekte, die mit Fragen der Identität und Emotion zusammenhängen. Insofern wäre es interessant, 
FERREIRA, M.V.; MARQUES-SCHÄFER, G.; BOLACIO FILHO, E. - DaF an öffentlichen Schulen

die Auswirkungen des Projekts auf die Identitätskonstruktion dieser Schüler*innen genauer zu beobachten, ebenso wie die erwähnten emotionalen Aspekte, die sich nicht nur auf das Lernen, sondern auch auf andere Lebensbereiche der Schüler*innen auswirken können, wie z.B. die Verbesserung der Arbeitsstimmung, der Motivation und der Freude am Sein und Lernen in der Schule.

Im Hinblick auf die theoretische Diskussion und die Elemente, die sich aus der Forschung ergeben haben, erkennen wir die Relevanz und den bahnbrechenden Charakter des Projekts in Bezug auf den Fremdsprachenunterricht in Rio de Janeiro. Es wäre wichtig, wenn weitere Studien über andere Aspekte des hier untersuchten Projekts durchgeführt werden könnten, um die Förderung des kulturellen Deutschlernens außerhalb des deutschsprachigen Raums besser zu erforschen.

\section{Anhans}

\section{Fragebogen}

\section{Liebe LehrerInnen,}

Wir führen eine Studie über den Deutschunterricht und die Unterrichtsmaterialien in der Grundschule durch. Ihre Teilnahme ist sehr wichtig für die Forschung. Bitte nehmen Sie sich circa 15 Minuten Zeit, um den folgenden Fragebogen auszufüllen. Mit dem Ausfüllen und Absenden geben Sie uns die Erlaubnis, die Daten für wissenschaftliche Zwecke zu verwenden. Ihre Identität wird gewahrt. Herzlichen Dank für Ihre Teilnahme. Wenn Sie mehr über die Umfrage wissen möchten, scheuen Sie sich nicht, uns zu kontaktieren.

Mit freundlichen Grüßen,

\section{Die AutorInnen}

\section{Fragen}

1. Wie lange arbeiten Sie schon in öffentlichen Schulen? Wie lange arbeiten Sie schon als Deutschlehrerin bzw. Deutschlehrer?

2. Wie würden Sie die Ziele des Deutschunterrichts an Ihrer Schule unter Berücksichtigung Ihres spezifischen Kontextes beschreiben? 
FERREIRA, M.V.; MARQUES-SCHÄFER, G.; BOLACIO FILHO, E. - DaF an öffentlichen Schulen

3. Im Jahr 2018 trafen die für das Projekt ausgewählten Lehrbücher nicht rechtzeitig ein. Das gesamte Material wurde von den Lehrern*innen erstellt. Welche sprachlichen Themen haben Sie in diesem Jahr mit Ihren Lernenden bearbeitet?

4. Welche kulturbezogenen Themen haben Sie gewählt?

5. Welche Aktivitäten behandelten diese kulturellen Aspekte (Klassenprojekte, Schulveranstaltungen, Besuche, Konzerte usw.)?

6. Wie können diese Aktivitäten nach Ihrer Einschätzung zur Entwicklung der interkulturellen Kompetenz der beteiligten Lernenden beitragen?

7. Wenn Sie an die Entwicklung der Kinder denken, wie können Sie aus Ihrer Erfahrung als Lehrer die Auswirkungen des Deutschunterrichts auf die Kinder Ihrer Schule in pädagogischer und soziokultureller Hinsicht beschreiben?

8. Und in kognitiver und emotionaler Hinsicht?

9. Bitte geben Sie hier bis zu 10 Adjektive an, die aus Ihren Erfahrungen den Deutschunterricht für Kinder an Ihrer Schule charakterisieren.

\section{Literaturverzeichnis}

AgUiAR, Ana R. R. A Educação Intercultural no entendimento da Diversidade na sala de aula de Lingua Estrangeira. Dissertation (Promotion in Didaktik der Fremdsprachen) Faculdade de Letras da Universidade do Porto, Porto, 2010. Verfügbar unter: https://repositorioaberto.up.pt/bitstream/10216/53883/2/tesedoutanaaguiar000120940.pdf (21/08/2021).

ALMEIDA, Ricardo L. T. Between the language instructor and the language educator: profiles of undergraduate students of English in pre-service teacher education. Ecos de Linguagem, v. 3, 101-123, 2014.

BRASIL. Lei de Diretrizes e Bases da Educação Nacional. Lei número 9394, 20 de dezembro de 1996.

BRASIL. Ministério da Educação. Base Nacional Comum Curricular. Brasília, 2017. Verfügbar unter: http://basenacionalcomum.mec.gov.br (01/03/2021).

BRASIL. Ministério da Educação. Base Nacional Comum Curricular. Secretaria de Educação Básica. Diretoria de Currículos e Educação Integral. Brasília: MEC, 2018.

BRUNSMEIER, Sonja. Interkulturelle kommunikative Kompetenz im Englischunterricht der Grundschule. Grundlagen, Erfahrungen, Perspektiven. Tübingen: Narr, 2016.

BYRAM, Michael. Teaching and Assessing Intercultural Communicative Competence. Clevedon: Multilingual Matters, 1997.

Chizzotti, Antonio. Pesquisa em ciências humanas e sociais. Petrópolis: Vozes, 2006.

DAY, Kelly. Ensino de língua estrangeira no Brasil: entre a escolha obrigatória e a obrigatoriedade voluntária. Revista Escrita, n. 15, 2012, 1-13. Verfügbar unter: https://www.maxwell.vrac.puc-rio.br/20850/20850.PDF (24/09/2021) 
FERREIRA, M.V.; MARQUES-SCHÄFER, G.; BOLACIO FILHO, E. - DaF an öffentlichen Schulen

DENZIN, Norma K.; LinCOLn, Yonna S. (Hrgs.). Handbook of qualitative research. 2. Ed. Califórnia: SAGE Publications, 2000.

ECKERT, Penelope. Linguistic variation as social practice. Oxford: Blackwell, 2000.

EDMUNDO, Eliana Santiago. O ensino de inglês na escola pública sob a perspectiva do letramento crítico. Masterarbeit - Setor de Ciências Humanas, Letras e Artes, Universidade Federal do Paraná, Curitiba, 2010.

EUROPARAT. Rat für kulturelle Zusammenarbeit. Gemeinsamer europäischer Referenzrahmen für Sprachen: lernen, lehren, beurteilen. Berlin, München, Wien, Zürich, New York: Langenscheidt, 2000.

FreIRE, Paulo. Educação e mudança. São Paulo: Editora Paz e Terra, 1979.

FREIRE, Paulo. Educação como prática da liberdade. Rio de Janeiro: Paz e Terra, 1999.

FreIRE, Paulo. Pedagogia da Autonomia: saberes necessários à prática da autonomia. Rio de Janeiro: Editora Paz e Terra, 2001.

FreIRE, Paulo. Pedagogia do oprimido. Rio de Janeiro: Paz e Terra, 2002.

FREIRE, Paulo. Pedagogia da tolerância. São Paulo: UNESP, 2005.

KNAPP, Ilona. Textkompetenzen beim Lehren und Lernen von modernen Fremdsprachen. In: BASSOLA, Péter et al. (Hrsg.). Zugänge zum Text (Szegediner Schriften zur germanistischen Linguistik 3). Frankfurt am Main: Lang, 2014, 127-150.

LEFFA, Vilson. O ensino de línguas estrangeiras no contexto nacional. Contexturas, APLIESP, n. 4, 13-24, 1999.

LEFFA, Vilson. Criação de bodes, carnavalização e cumplicidade: considerações sobre o fracasso da LE na escola pública. In: LIMA, Cândido D. (Hrsg.). Inglês em escolas públicas não funciona? Uma questão, múltiplos olhares. São Paulo: Parábola, 2011, 15-32.

LEFFA, Vilson; IRALA, Valesca (Hrsg.). Uma espiadinha na sala de aula: ensinando línguas adicionais no Brasil. Pelotas: Educat, 2014.

LiMA, Diógenes C. (Hrsg.). Inglês em escolas públicas não funciona? Uma questão, múltiplos olhares. São Paulo: Parábola, 2011.

MAtTos, Andrea O. Novos letramentos, ensino de língua estrangeira e o papel da escola pública no século XXI. In: JORDÃO, C. (Hrsg.). Letramentos e Multiletramentos no Ensino de Línguas e Literaturas. Revista X, v. 1, 33-47, 2011.

MARQUES-SChÄFER, Gabriela; STANKE, Roberta C.S.F. Didactic, Linguistic and Educational Issues in Intercultural Studies: an Introduction. In: MARQUES-SCHÄFER, G.; STANKE, R.C.S.F. (Hrsg.). DaF e aprendizagem intercultural / DaF und interkulturelles Lernen. GiF:on - Giessener Fremdsprachendidaktik: online 17. Giessen University Library Publications, 2021, 1-12.

MENEZES DE SOUZA, Lynn Mario. T.; MONTE MÓR, Walkiria M. Orientações curriculares para o ensino médio: linguagens, códigos e suas tecnologias - conhecimentos de línguas estrangeiras. Brasília: Ministério da Educação / Secretaria de Educação Básica, 2006. Verfügbar unter: http://portal.mec.gov.br/seb/arquivos/pdf/book_volume_01_internet.pdf (06/02/2021).

MoITA LoPES, Luiz Paulo. Pesquisa interpretativa em linguística aplicada: a linguagem como condição e solução. Delta, v. 10, n. 2, 329-338, 1994.

MULIK, Katia Bruginski. O ensino de língua estrangeira no contexto brasileiro: um passeio pela história. Crátilo: Revista de Estudos Linguísticos e Literários, v. 5, n. 1, 14-22, 2012.

OlabuenAGA, José Ignácio R.; IsPIZUA, Maria Antonia. La descodificacion de la vida cotidiana: metodos de investigacion cualitativa. Bilbao, Universidad de Deusto, 1989.

OliveIRA, Renilson Santos. Linha do tempo da didática das línguas estrangeiras no Brasil. Non Plus, n. 7, 2015.

PARANÁ (Governo do Estado). Diretrizes Curriculares da Educação Básica Língua Estrangeira Moderna. Governo do Estado do Paraná, 2008. Verfügbar unter: 
FERREIRA, M.V.; MARQUES-SCHÄFER, G.; BOLACIO FILHO, E. - DaF an öffentlichen Schulen

http://www.educadores.diaadia.pr.gov.br/arquivos/File/diretrizes/dce_lem.pdf $(21 / 08 / 2021)$.

RiO DE JANEIRO (Município). "Rio, Criança Global implanta ensino de Inglês desde o $1^{\circ}$ ano na rede municipal". Página Eletrônica da Secretaria Municipal de Educação (SME), set. de 2010. Verfügbar unter: http://www.rio.rj.gov.br/web/sme/exibeconteudo?articleid=1100300 (21/08/2021).

RiO DE JANEIRO (Município). Parecer "N" $n^{\circ} 1 / 2018$. Aprova o funcionamento de escolas bilíngues inglesa, francesa e espanhola, e autoriza, em caráter experimental, a escola bilíngue alemã nas Unidades Escolares da Rede Pública do Sistema Municipal de Ensino do Rio de Janeiro e dá outras providências. In: Diário Oficial do Município do Rio de Janeiro, ano XXXII, n. 53, 59-60, 5 jun. 2018a.

RIO DE JANEIRO (Município). Parecer "N" $\mathrm{n}^{\circ} 1 / 2018$. Aprova o funcionamento de escolas bilíngues inglesa, francesa e espanhola, e autoriza, em caráter experimental, a escola bilíngue alemã nas Unidades Escolares da Rede Pública do Sistema Municipal de Ensino do Rio de Janeiro e dá outras providências. In: Diário Oficial do Município do Rio de Janeiro, ano XXXII, n. 54, 44, 6 jun. 2018b.

RoJo, Roxane Helena R. Pedagogia dos multiletramentos: diversidade cultural e de linguagens na escola. In: RoJo, Roxane Helena R.; MouRA, Eduardo (Hrsg.). Multiletramentos na escola. São Paulo: Parábola Editorial, 2012, 11-32.

RUSCHEINSKY, Aloísio. Cidadania e construção do conhecimento ante a expressão de interesses. In: LAMPERT, Ernani. (Hrsg.) Educação para a cidadania: gênero, etnia, políticas educacionais, competência docente/discente. Porto Alegre: Fundação Universidade do Rio Grande/Editora Sulina, 1999, 13-32.

SANTOS, Rodolfo; IFA, Sérgio. O letramento crítico e o ensino de inglês: reflexões sobre a prática do professor em formação continuada. The Especialist, v. 34, n. 1, 1-23, 2013.

SCHLATTER, Margarete. O ensino de leitura em língua estrangeira na escola: uma proposta de letramento. Calidoscópio, v. 7, n. 1, 11-23, 2009.

SCHLATTER, Margarete.; GARCEZ, Pedro M. Linguas adicionais na escola: aprendizagens colaborativas em inglês. Erechim: Edelbra, 2012.

SCHMÖLZER-EIBINGER, Sabine. Lernen in der Zweitsprache. Grundlagen und Verfahren der Förderung von Textkompetenz in mehrsprachigen Klassen. Tübingen: Gunter Narr Verlag, 2008.

SoARES, Magda. Letramento: um tema em três gêneros. Belo Horizonte: Autêntica, 2009.

Recebido em 29 de abril de 2021

Aceito em 10 de setembro de 2021

Pandaemonium, São Paulo, v. 25, n. 45, jan.-abr. 2022, p. 1-28 